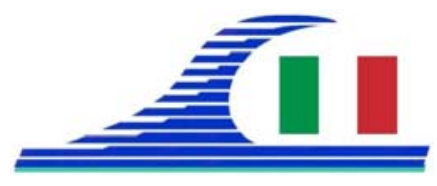

Conférence Méditerranéenne Côtière et Maritime EDITION 3, FERRARA, ITALIA (2015)

Coastal and Maritime Mediterranean Conference

Disponible en ligne - http://www.paralia.fr - Available online

\title{
Estimation du tassement de la plateforme de l'extension du port d'Alger renforcée par des colonnes ballastées
}

\author{
Sadok BENMEBAREK ${ }^{1}$, Zakaria BOURAOUI ${ }^{1}$, \\ Naima BENMEBAREK ${ }^{1}$, Fatima DERIAS ${ }^{2}$
}

1. Laboratoire de recherche MN2I2S, Université de Biskra, Algérie.

Sadok_benmebarek@yahoo.com

2. Organisme National de Contrôle Technique des Travaux Publics, Algérie.

fatider@gmail.com

\section{Résumé :}

La présente communication s'intéresse à l'estimation du tassement du cas de l'extension de la plateforme du port d'Alger en cours du traitement par des colonnes ballastées. Différentes modélisations numériques ont été effectuées à l'aide du logiciel PLAXIS. Les résultats numériques sont comparés avec ceux des différentes approches analytiques disponibles dans la littérature en soulignant les avantages des approches numériques.

Mots-clés : Travaux maritimes, Modélisation numérique, Colonnes ballastées, Tassement.

\section{Introduction}

Les colonnes ballastées constituent une technique intéressante pour l'amélioration en place des sols compressibles. Le développement important de cette technologie de renforcement a conduit de nombreux auteurs à proposer des méthodes de calcul et à élaborer des abaques de dimensionnement (BAUMANN \& BAUER, 1974 ; PRIEBE, 1995 ; ...). Par ailleurs, la méthode observationnelle est très souvent employée dans tout projet important d'amélioration de sol (ALLAGNAT, 2005). La comparaison des tassements observés et calculés montrent généralement des différences notables (DHOUIB \& BLONDEAU, 2005). La présente communication s'intéresse à l'estimation du tassement du cas de l'extension de la plateforme du port d'Alger en cours du traitement par des colonnes ballastées. Différentes modélisations numériques à l'aide du logiciel PLAXIS 2D en éléments finis ont été effectuées en testant différents modèles géométriques.

\section{Présentation du projet}

Le projet concerne le traitement de la plateforme du port d'Alger potentiellement liquéfiable et déformable afin de supporter une surcharge d'exploitation de $5.5 \mathrm{t} / \mathrm{m}^{2}$ sur une largeur de $16.38 \mathrm{~m}$ (7 rangs de conteneurs) avec limitation du tassement à $5 \mathrm{~cm}$. Le programme de reconnaissance géotechnique effectué en 2013 a révélé la présence d’une couche compressible de sables limoneux vaseux d'épaisseur comprise entre 10 et $16 \mathrm{~m}$ 
Côtes méditerranéennes menacées :

Risques et défis dans le contexte du changement climatique

reposant sur une couche d'argile marneuse. La nappe phréatique a été identifiée à une profondeur d'environ $2 \mathrm{~m}$ par rapport à la surface du sol.

Le tableau 1 récapitule les paramètres du sol, du matelas et des colonnes ballastées retenus pour l'estimation du tassement. En absence des résultats des essais, les paramètres du ballast du matelas et des colonnes ballastées ont été choisis à partir de la littérature. Compte tenu des caractéristiques du sol de site, la solution retenue est l'amélioration du sol compressible par des colonnes ballastées par voie humide disposées selon une maille de distribution hexagonale avec une séparation entre axes variable en fonction de la variation de la couche compressible et les résultats des planches d'essais. Le contrôle de réalisation des colonnes a été assuré par des essais de chargement des colonnes, essais au pénétromètre statique CPT entre et au sein des colonnes en plus l'enregistrement de tous les paramètres d'exécution de la machine.

Tableau 1. Paramètres du sol, du matelas et des colonnes.

\begin{tabular}{lllllll}
\hline & $\gamma_{\text {sat }}\left(\mathbf{k N} / \mathbf{m}^{3}\right)$ & $\gamma_{d}\left(\mathbf{k N} / \mathbf{m}^{3}\right)$ & $\boldsymbol{C}^{\prime}(\mathbf{k P a})$ & $\boldsymbol{\varphi}^{\prime}\left({ }^{\circ}\right)$ & $\boldsymbol{E}^{\prime}(\mathbf{M P a})$ & $\boldsymbol{v}$ \\
\hline Sables vaseux & 19.62 & 16.68 & 0 & 32 & 5.50 & 0.27 \\
Argile marneuse & 19.31 & 15.20 & 0 & & & \\
Matelas & 23.00 & 20.00 & 0 & 40 & 60.00 & 0.25 \\
Colonnes ballastées & 23.00 & 20.00 & 0 & 40 & 60.00 & 0.25 \\
\hline
\end{tabular}

\section{Estimation de l'amélioration du tassement}

Plusieurs auteurs ont étudié les tassements des ouvrages sur sols traités par colonnes ballastées, en termes de facteur de réduction des tassements. Les approches analytiques les plus utilisées dans la pratique courante sont la méthode de PRIEBE (1995), la méthode d’homogénéisation simplifiée, la méthode de BAUMANN et BAUER (1974) et la règle T4 de Ménard (GAMBIN, 1984). L’hétérogénéité des sites traités et la variété des techniques de mise en œuvre offrent aussi un domaine très favorable à l'utilisation des méthodes numériques (éléments finis et différences finies).

Tableau 2. Comparaison des tassements calculés par des approches analytiques.

\begin{tabular}{ll}
\hline Méthodes & Tassement (cm) \\
\hline Avant traitement & 15.0 \\
Méthode de PRIEBE (1995) & 7.2 \\
Méthode d'homogénéisation simplifiée & 5.6 \\
Méthode de BAUMANN et BAUER (1974) & 4.0 \\
Règle T4 de Ménard (GAMBIN, 1984) & 1.1 \\
\hline
\end{tabular}

Les résultats de calcul de comparaison de ces approches analytiques effectués sur le présent projet, récapitulés dans le tableau 2, montrent des résultats diversifiés du tassement après traitement variant de 1.1 à $7.2 \mathrm{~cm}$. La règle T4 de Ménard a donné la valeur la plus faible de tassement $(1.1 \mathrm{~cm})$ nettement loin des résultats des autres 
méthodes. Cette règle est plutôt recommandée pour les colonnes courtes sujet de faible tassement. En conséquence, des modélisations numériques à l’aide du logiciel PLAXIS 2D ont été effectuées par deux approches de déformation (figure 1).

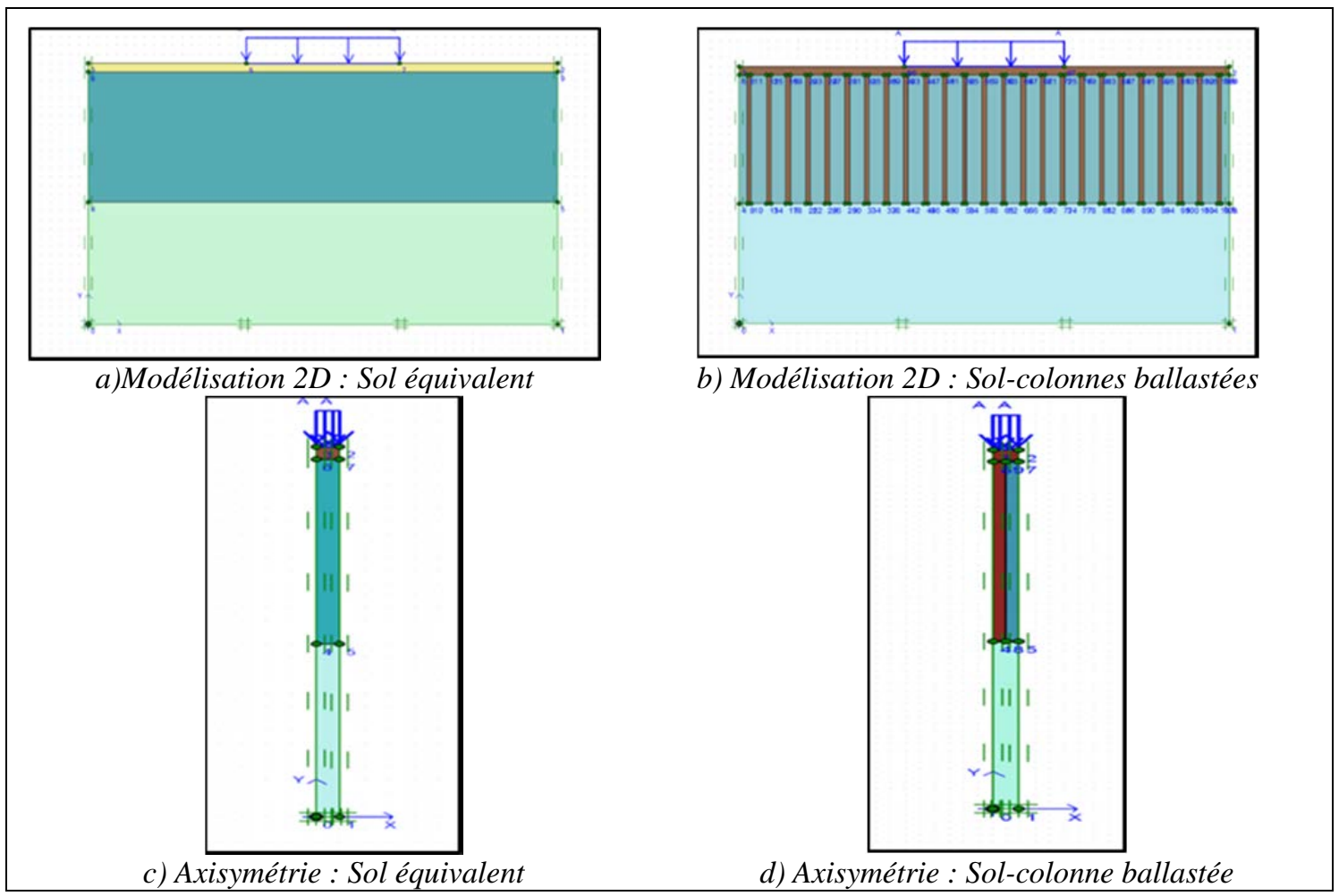

Figure 1. Modèle géométrique aux éléments finis.

Dans la première approche, le comportement de la zone traitée est approché par une déformation plane en considérant d'une part un sol équivalent (figure 1-a) et d'autre part un groupe de colonnes (figure 1-b). Dans la seconde avec le sol adjacent le comportement de l'ensemble est représenté par le comportement axisymétrique d'une unité de colonne ballastée avec son sol entourant. Egalement dans cette approche, la zone traitée est remplacée d'une part par un sol équivalent (figure 1-c) et d'autre part en respectant la géométrie du sol et de la colonne (figure 1-d). Le tableau 3 récapitule les résultats de calcul à l'aide du logiciel PLAXIS 2D du tassement en considérant les différents modèles géométriques.

A la lumière des résultats obtenus, on peut constater :

- Avant traitement du sol, une bonne concordance est notée entre déformation plane et déformation axisymétrique,

- Après traitement du sol, le modèle le plus simple "sol équivalent" donne des valeurs de tassements relativement proches du modèle sol-colonne,

- Les résultats des différentes approches de modélisation numérique concordent bien avec la méthode de Priebe et la méthode d'homogénéisation simplifiée. Cependant 
Côtes méditerranéennes menacées :

Risques et défis dans le contexte du changement climatique

une divergence fortement notable est remarquée en comparant avec la règle T4 de Ménard.

Tableau 3. Comparaison des tassements calculés par PLAXIS.

\begin{tabular}{llll}
\hline \multirow{2}{*}{ Modèle } & & Matériau & Tassement $(\mathbf{c m})$ \\
\hline \multirow{2}{*}{$\begin{array}{l}\text { Avant } \\
\text { traitement }\end{array}$} & Déformation plane & \multirow{2}{*}{ Sol naturel } & 14.5 \\
\cline { 2 - 4 } $\begin{array}{l}\text { Après } \\
\text { traitement }\end{array}$ & Déformation axisymétrique & & 14.3 \\
\cline { 2 - 4 } & Déformation plane & Sol équivalent & 5.1 \\
\cline { 2 - 4 } & Déformation axisymétrique & Sol équivalent & 6.6 \\
\cline { 3 - 4 } & & Sol-colonne & 6.6 \\
\hline
\end{tabular}

\section{Conclusions}

Une étude comparative utilisant les approches analytiques et numérique en utilisant le logiciel PLAXIS en éléments finis a été effectuée afin d'évaluer l'amélioration du tassement après traitement de la plateforme à conteneurs du port d'Alger par des colonnes ballastées. A partir des résultats de cette étude comparative, nous pouvons tirer les conclusions suivantes :

- Les méthodes analytiques recommandées pour le cas de traitement des sols par colonnes ballastées sous charge répartie ont donné des tassements dispersés pour le présent projet ;

- Les différentes approches numériques à savoir en déformation plane et déformation axisymétrique avec sol équivalent et sol-colonne ont montré des résultats de tassement relativement serrés et comparables aux résultats des méthodes de Priebe et d’homogénéisation simplifiée ;

- L’approche numérique peut présenter des avantages particulièrement pour des configurations complexes.

\section{Références bibliographiques}

ALLAGNAT D. (2005). La méthode observationnelle pour le dimensionnement interactif des ouvrages. Presses de l'ENPC, 127 p.

BAUMANN V., BAUER G.E.A., (1974). The performance of foundations on various soils stabilized by the Vibrocompaction process. Revue canadienne de géotechnique, Vol. 11(4), pp 509-530.

DHOUIB A., BLONDEAU F. (2005). Colonnes ballastées. Techniques de mise en ouvre, domaines d'application, comportement, justification, contrôle, axes de recherche et de développement, Presses des Ponts, 264 p.

GAMBIN M. (1984). Puits ballastés à la Seyne-sur-Mer. Actes du colloque International: Renforcement en place des sols et des roches. Paris, Vol. 1, pp 139-144. PRIEBE H. (1995). The design of vibro-replacement. Reprinted from Ground Enginering. Keller Grundbau GmbH, December 1995, Technical paper 12-61E, 16 p. 